\title{
Mathematical Modeling: Zoonotic Strength of Infectivity on COVID-19
}

\author{
R. Kandasamy ${ }^{1} \cdot$ Sisai Bekele $^{1} \cdot$ Tolossa Belete $^{1}$
}

Received: 2 November 2021/Revised: 2 November 2021 / Accepted: 8 November 2021/Published online: 26 December 2021

(C) The National Academy of Sciences, India 2021

\begin{abstract}
Coronavirus 2019 (COVID-19) is an emerging contagious disease that has led to the global epidemic and is caused by severe acute respiratory syndrome, Coronavirus-2 (SARS-CoV-2). Zoonotic is an infection that is transmitted from animals to humans. Significantly changing animal infection is the best amplifying mask of pathogens. The power of animal infection is a contagious virus that usually spreads from vertebrate animals to humans and vice versa. The physical health of an animal depends on the normal vulnerability of its population and the change in actual animal health against infection over time, and is assessed based on previous circumstances in the population. After compiling the effect of virulence of the animal infection on the size of the susceptible, exposed and infected subjects, an investigation was made using MAPLE 18 with RK Fehlberg technique. This study discusses the stimulation of animal influences on the novel coronavirus infection that is reliable to be infectious. It analyzes all incoming and outgoing air passengers worldwide and in the country is keen on spreading the abnormally degenerate coronavirus. The zoonotic strength was applied to coronavirus infection and the rates of SEIR individuals terminated by secondary coronavirus infection were estimated. The transmission ability of coronavirus
\end{abstract}

Sisai Bekele

sisai@slu.edu.et

R. Kandasamy

future990@gmail.com

Tolossa Belete

tolossadadI@yahoo.com

1 College of Natural Science, Salale University, Fiche, Oromia Region, Ethiopia infection is associated with a lack of the perceived respiratory system in conservative people and global warming.

Keywords Coronavirus - Susceptible $\cdot$ Exposed Infected peoples $\cdot$ Zoonotic $\cdot$ Global reheating

\section{Introduction}

It has been more than six months since experts were aware of the onset of a highly contagious disease known as COVID-19. Since then, people around the world have learned about the devastating effects of the disease that can be transmitted from animals to humans. Approximately $60 \%$ of known infectious diseases and $75 \%$ of emerging or emerging human diseases are from animals. SARS-CoV-2 is the latest additions to the seven coronaviruses are found in humans, and experts say they all come from bats, rodents or pets. Animals can occasionally transmit hurtful microorganisms that can blowout to people and basis illness, these are recognized as quixotic viruses or zoo noses. Zoo noses include a bulky proportion of all recently recognized transmittable viruses along with current transferable viruses. Antimicrobial struggle in human pathogens is additional chief civic well-being risk which is moderately squeezed by practice of antibiotics in animal farming and agronomy, [1,2].

Some zoonotic viruses are friendly, but many Lyme viruses are dangerous. But the coronavirus like COVID-19 can be very dangerous to humans. The main theory about the coronavirus outbreak is that the virus will end in people at a wet market in Wuhan, China, at the end of 2019. It is not known how active the disease is from new flocks to natural markets and to humans, [3, 4]. International and domestic air passengers, arrivals and departures have been 
identified as key factors in the prevention of the disease. Spread the strength of coronavirus infection in different countries. The transmission of the coronavirus from the air and natural reservoirs is a highly anticipated event, and zoonotic transmission of the coronavirus is a more likely event. There is a growing awareness of the incidence of animal coronavirus [5-8]. These viruses, which are transmitted from animal to human through direct and indirect interactions, are known as zoonotic viruses or zoonosis, and COVID-19 is a one of them.

COVID-19 is one of the worst animal diseases. But not the first disease Ebola sar, Smears, HIV, Lyme disease, Rift valley and Lassa fever. In the past century, we have seen at least six outbreaks of the novel coronavirus, [9-12].

In an analogous discovery available approximately in Korea, the outlays had been equivalent, with subordinate contagions in 0.55 segment of all interactions and 7.6 magnitudes of domestic persons [13]. Collections of occasions have moreover been noticeable ensuing family, work, or social crowds the residence nearby, isolated interaction can perform [14]. As an example, epidemiologic estimation of a collection of occasions in the country of Illinois established possibly spread through two household assemblies at which shared meals applied to be inspired squeezes had been joint, and continued face-toface discussions had been replaced with suggestive persons who had been advanced experienced to have COVID-19 [15]. The hazard of spread with superior tilted interaction is now not agreeably connected and is possible truncated. Although the industrialized nations have been skilled to restrain fresh bursts of zoonotic infectious, many resources-controlled and some few countries have not been efficient to react adequately.

During the COVID-19 outbreak, although there were previously outbreaks of animals after four individual reports from the seafood wholesale market were reported. But all other transmission is likely from person to person (Figs. 1 and 2).

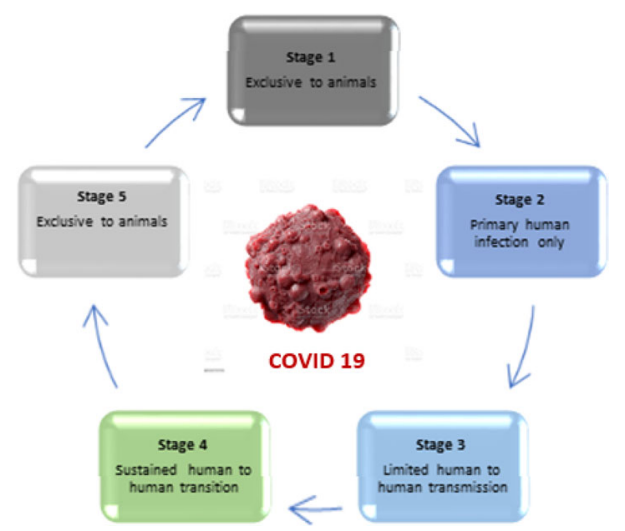

Fig. 1 Zoonotic strength of infectivity on COVID-19
This mode of transmission is so active that it can fly to another country within a few days. In the recent outbreak, the human-to-human transmission has increased significantly due to the annual celebrations in China at a time of massive increase in mass movement. Transmission of infection from person to person can happen in a number of ways. This can be from coughing or sneezing in public transport areas, restaurants, and other public places (restrooms, elevators, bus stops). As in the past with other animal conditions, the spread of the new SARS-CoV-2 coronavirus is now important. The current and past origins of coronavirus bats, such as SARS-COV and MERS-COV, are concerned in animal-human interactions, with concerns in the future of new pandemics in China and abroad.

Due to this research, we are penetrating about the impression of zoonotic strength of infectivity depends on coronavirus susceptible, exposed, and infected populations are repossessed with the assistance of MAPLE 18. The proposal of widespread differential equations is expressed to the aid of restrained individuals, uncovered individuals, characteristic polluted persons are foreseeable with the support of employing R. K. Fehlberg useful with the backing of MAPLE 18. Pedals on astonishing modifiable herbal abundances on the vulnerable, unprotected, and diseased populations are stated by way of charts and the new enhancements are related to the smart description, $[14,15]$.

\section{Mathematical Modeling and Analysis}

The epidemic of the coronavirus COVID-19 has reserved the exists of ample thousand global and sealed-out many nations and areas, with however impulsive global significances. This systematic research relies upon at the susceptible, unprotected, and diseased populations of organization tasks. It's far authenticated how public activities which includes isolation, regional lockdowns, confinement, and global public suitability attentiveness, have a motivation at the infested forms' parameters, which could inside the expiry substitute the humanity incidentals and bursting of presence grimy events past an out-of-date, in the physical international. To propose the countless prospect of researcher's, examine to rheostat spreading established coronavirus viruses, a speedy foundation on herbal constructions scheming is in addition disbursed and the maple coding for the clinical researches are appointed in this effort. 
Fig. 2 Cyclic transmission of Zoonotic infectivity, COVID-19

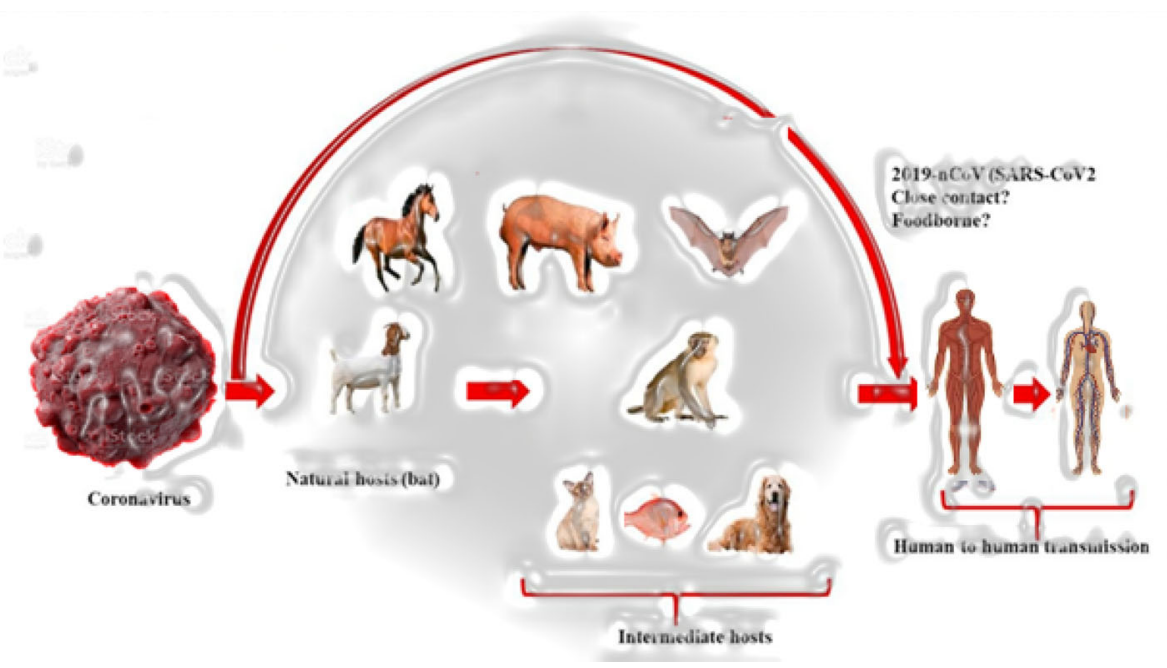

\subsection{Control Panel: Definition of the 2019 New Corona Virus (2019-nCoV)}

2019-nCoV case definitions differ depending on the context in which they are used.

\subsection{Case definition of the Chinese Centers for Disease Control and Prevention (CDC)}

A suspected or possible case is defined as a case that meets: (1) three clinical criteria or (2) two clinical and one epidemiological criteria. Clinical criteria include fever; Radiation evidence of pneumonia or acute respiratory distress syndrome. And low or normal white blood cell count or low white blood cell count Epidemiological criteria: live in Wuhan or travel to Wuhan within 14 days before the onset of symptoms; Contact a patient with a fever and symptoms of a respiratory infection within 14 days of the onset. And links to confirmed cases or groups of suspects.

The first confirmed case definitions in the province were suspected or possible, with viral nucleic acids tested at the CDC in the city and the province's CDC for the second case and the case. All along, the definition is a suspected or possible case with viral nucleic acid detection at the urban CDC.

\subsection{Case Definitions used in the Case Output Model in this Study}

We define cases as individuals with symptoms that can be detected by temperature checks at international borders, or people with serious illness requiring hospitalization or both, including a history of travel to Wuhan.

The current study uses SEIR [14] as a common channel model in epidemiological studies. Demonstrate how disease progresses through four interactions: risk, disclosure, contact and disappearance. It depends on three variables: the rate of exposure, exposure, recovery from infectious disease, and infection. The last two were inversely related to latency and infection duration, respectively. Pollution generated in the infectious zone was aimed at the lease of a clear classification framework. We think that people in the region recognize that they are aware of a number of these issues, $S_{p}, E_{p}, I_{p}, L_{1}, L_{2}, L_{3}, L_{4}, D_{1}, D_{2}$ and $R_{0}$. For clarity, we believe that the populations in that zone are always allocated equally (can be developed by separating some regions into a series of shorter regions of the same nature). We accept that reincarnation is ineffective. We do not monitor individual movements between countries. We use the following mathematical design for Susceptible-Exposed-Infectious-Recovered (SEIR) later to calculate the Wuhan-infected virus as expected in December 2019 $[14,15]$ :

$$
\begin{aligned}
\frac{d S(t)}{d t}= & -\frac{S(t)}{N}\left(\frac{R_{0}}{D_{1}} I(t)+z_{0}\right)+L_{1}+L_{2} \\
& -\left(\frac{L_{3}}{N}+\frac{L_{4}}{N}\right) S(t) \\
\frac{d E(t)}{d t}= & \frac{S(t)}{N}\left(\frac{R_{0}}{D_{1}} I(t)+z_{0}\right)-\frac{E(t)}{D_{2}}-\left(\frac{L_{3}}{N}+\frac{L_{4}}{N}\right) E(t) \\
\frac{d I(t)}{d t}= & \frac{E(t)}{D_{2}}-\frac{I(t)}{D_{1}}-\left(\frac{L_{3}}{N}+\frac{L_{4}}{N}\right) I(t)
\end{aligned}
$$

with boundary conditions 


$$
\begin{aligned}
S(0)= & S_{0}, u(0) \\
= & -\frac{S_{0}}{N}\left(\frac{R_{0}}{D_{1}} I(0)+z_{0}\right)+L_{1}+L_{2} \\
& -\left(\frac{L_{3}}{N}+\frac{L_{4}}{N}\right) S_{0}, E(0) \\
= & 0, I(0)=0 \text { when } t=0 ; \\
u(l)= & 1, E(l)=1, I(l)=1 \text { as } t \rightarrow l ;
\end{aligned}
$$

Assume that $E=E_{0}, I=I_{0}$ when $\mathrm{t}=0 ; E=E_{l}, I=I_{l}$ as $\quad t \rightarrow l$; with $E(t)\left(E_{0}-E_{l}\right)=\left(E-E_{l}\right)$, $I(t)\left(I_{0}-I_{l}\right)=\left(I-I_{l}\right)$, where $S_{0}$-Newly birth people, $E_{0}$-Exposed people before coronavirus infection, $I_{0}$ Infected people before coronavirus infection, $E_{l}$-Exposed people from coronavirus infection, $I_{l}$-Infected people from coronavirus infection. $L_{1}$-International inbound air passengers, $L_{2}$-Domestic inbound passengers, $L_{3}-$ Outbound International passengers, $L_{4}$-Domestic outbound passengers, $D_{1}$-Infectious period, $D_{2}-$ Mean latent, $R_{0}$-Essential generative quantity, $Z_{0}$ - the zoonotic strength of infectivity, $N$-Total number peoples. $S(t)$-Number of susceptible peoples, $E(t)-$ Number of exposed peoples, $I(t)$-Number of infected peoples, $R(t)$-Number of detached entities at time $\mathrm{t}$.

\section{Numerical Analysis}

Analytical scheme is appointed to show the natural troubles of the inclined-exposed-infected individuals from coronavirus over the existing-day dispute. It is admirable to authenticate this mathematical mechanism and the scientific computational machine is extensively to resolve multiplicity, bodily or geometrically. We famed the computational algorithm handles numerical software usually to clear up numerous complexities. But we ought to beforehand in notice that every one of the coding software program technique has been confirming the analytical success anciently. The medical research coding structures are controlled to forecast the issue. Besides, numerical mechanisms are activated to assume the willing, unprotected, and diseased citizens for practical efforts.

Equations (8), (9) are remodeled as.

$$
\begin{gathered}
\operatorname{diff}(f(\eta), \eta)=u(\eta), \operatorname{diff}(u(\eta), \eta)=v(\eta), \operatorname{diff}(\theta(\eta), \eta) \\
=p(\eta), \operatorname{diff}(\varphi(\eta), \eta)=h(\eta)
\end{gathered}
$$

$$
\begin{aligned}
\operatorname{diff}( & \left.v(\eta), \eta)+\frac{1}{N}\right)( \\
& -\frac{1}{N}\left(\left(-\frac{s(\eta)}{N}\left(\left(\frac{R 0}{D 1}\right) \cdot i(\eta)+z 0\right)+L 1+L 2-\left(\frac{L 3}{N}\right.\right.\right. \\
& \left.\left.\left.+\frac{L 4}{N}\right) \cdot s(\eta)\right)\right) \cdot\left(\frac{R 0}{D 1}\right) \cdot\left(\frac{e(\eta)}{D 2}-\frac{i(\eta)}{D 1}-\left(\frac{L 3}{N}+\frac{L 4}{N}\right)\right. \\
& i(\eta)))-\left(\frac{L 3}{N}+\frac{L 4}{N}\right) \cdot\left(-\frac{s(\eta)}{N}\left(\left(\frac{R 0}{D 1}\right) \cdot i(\eta)+z 0\right)+L 1\right. \\
& \left.\left.+L 2-\left(\frac{L 3}{N}+\frac{L 4}{N}\right) \cdot s(\eta)\right)\right) \cdot\left(\frac{R 0}{D 1}\right) \\
& +\left(\frac { 1 } { D 2 } \left(\left(\frac{s(\eta)}{N}\left(\left(\frac{R 0}{D 1}\right) \cdot i(\eta)+z 0\right)-\frac{e(\eta)}{D 2}-\left(\frac{L 3}{N}\right.\right.\right.\right. \\
& \left.\left.\left.+\frac{L 4}{N}\right) \cdot e(\eta)\right)\right)-\left(\frac{1}{D 1}+\frac{L 3}{N}+\frac{L 4}{N}\right) \cdot\left(\frac{e(\eta)}{D 2}-\frac{i(\eta)}{D 1}\right. \\
& \left.\left.-\left(\frac{L 3}{N}+\frac{L 4}{N}\right) \cdot i(\eta)\right)\right)+\left(\frac{L 3}{N}+\frac{L 4}{N}\right) \cdot( \\
& -\frac{1}{N}\left(\left(-\frac{s(\eta)}{N}\left(\left(\frac{R 0}{D 1}\right) \cdot i(\eta)+z 0\right)+L 1+L 2-\left(\frac{L 3}{N}\right.\right.\right. \\
& \left.\left.\left.+\frac{L 4}{N}\right) \cdot s(\eta)\right)\right) \cdot\left(\frac{R 0}{D 1}\right) \cdot\left(\frac{e(\eta)}{D 2}-\frac{i(\eta)}{D 1}-\left(\frac{L 3}{N}+\frac{L 4}{N}\right)\right. \\
& . i(\eta)))-\left(\frac{L 3}{N}+\frac{L 4}{N}\right) \cdot\left(-\frac{s(\eta)}{N}\left(\left(\frac{R 0}{D 1}\right) \cdot i(\eta)+z 0\right)+L 1\right. \\
& \left.+L 2-\left(\frac{L 3}{N}+\frac{L 4}{N}\right) \cdot(\eta)\right)=0,
\end{aligned}
$$

$$
\begin{aligned}
& \operatorname{diff}(h(\eta), \eta) \\
&- \frac{\left(\frac{s(\eta)}{N}\left(\left(\frac{R 0}{D 1}\right) \cdot i(\eta)+z 0\right)-\frac{e(\eta)}{D 2}-\left(\frac{L 3}{N}+\frac{L 4}{N}\right) \cdot e(\eta)\right)}{D 2} \\
&+\left(\frac{1}{D 1}+\frac{L 3}{N}+\frac{L 4}{N}\right) \cdot\left(\frac{e(\eta)}{D 2}-\frac{i(\eta)}{D 1}-\left(\frac{L 3}{N}+\frac{L 4}{N}\right) \cdot i(\eta)\right) \\
&=0
\end{aligned}
$$

$$
\begin{aligned}
\operatorname{diff} & (p(\eta), \eta) \\
& -\left(\frac { 1 } { N } \left(\left(-\frac{s(\eta)}{N}\left(\left(\frac{R 0}{D 1}\right) \cdot i(\eta)+z 0\right)+L 1+L 2-\left(\frac{L 3}{N}\right.\right.\right.\right. \\
& \left.\left.\left.\left.+\frac{L 4}{N}\right) \cdot s(\eta)\right)\right)\right) \cdot\left(( \frac { R 0 } { D 1 } ) \cdot \left(\frac{e(\eta)}{D 2}-\frac{i(\eta)}{D 1}-\left(\frac{L 3}{N}+\frac{L 4}{N}\right)\right.\right. \\
& . i(\eta)))+\left(\frac{1}{D 2}+\frac{L 3}{N}+\frac{L 4}{N}\right) \cdot\left(\frac{s(\eta)}{N}\left(\left(\frac{R 0}{D 1}\right) \cdot i(\eta)+z 0\right)\right. \\
& \left.-\frac{e(\eta)}{D 2}-\left(\frac{L 3}{N}+\frac{L 4}{N}\right) \cdot e(\eta)\right)=0, \\
I C & :=\{f(0)=0, u(0)=A, \theta(0)=1, \varphi(0)=1, v(0)=\alpha, p(0)=\tau, \\
& h(0)=\varsigma\} \\
B C & :=\{u(\mathrm{~L})=\mathrm{I}, \theta(\mathrm{L})=0, \varphi(L)=0\}
\end{aligned}
$$

$A:=\left(-\frac{S 0}{N}\left(\frac{R 0}{D 1}+Z 0\right)+L 1+L 2-\left(\frac{L 3}{N}+\frac{L 4}{N}\right) \cdot S 0\right)$
Expressed $\alpha, \tau$, and $\varsigma$ to be associated with weak, unprotected and infectious human coronavirus, the Runge- 


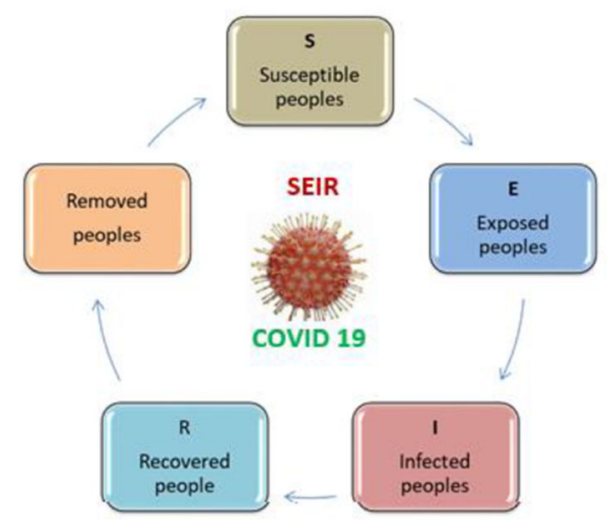

Fig. 3 SEIR Mathematical model

Kutta-Fehlberg technique, which was underestimated in determining the limit problem Dsolve had. The figure consisted of MAPLE 18. Expect an effort to approve $u$ '(0), E' (0) and I '(0) $\alpha, \tau$ and $\varsigma$ suggest that $\mathrm{v}(0)=\alpha, \mathrm{p}$ $(0)=\tau$ and $\mathrm{h}(0)=\varsigma$ with a link of trial and error. The cipher file examines a collection of vulnerable, unprotected and diseased communities of coronavirus infection.

\section{Results and Discussions}

A series of infected peoples who have been contagious and have come into contact with humans due to coronavirus have been investigated numerically alongside the circumstances. The project for Eqs. (6)-(8) cannot be logically edited and the mathematically responsive explanation of the situation is expected (9) to control the very important MAPLE 18. This software uses the Runge-Kutta-Fehlberg method with a validated technique to mathematically verify boundary rate problems (Fig. 3).

The effect of the virulence of primary zoonotic based on the COVID-19 infection has been successfully described. It is expected to control the spread of people who are likely to be exposed and infected with the COVID-19 and to be used for human safety, Figs. 4, 5, 6, 7 and 8. The key zoonotic
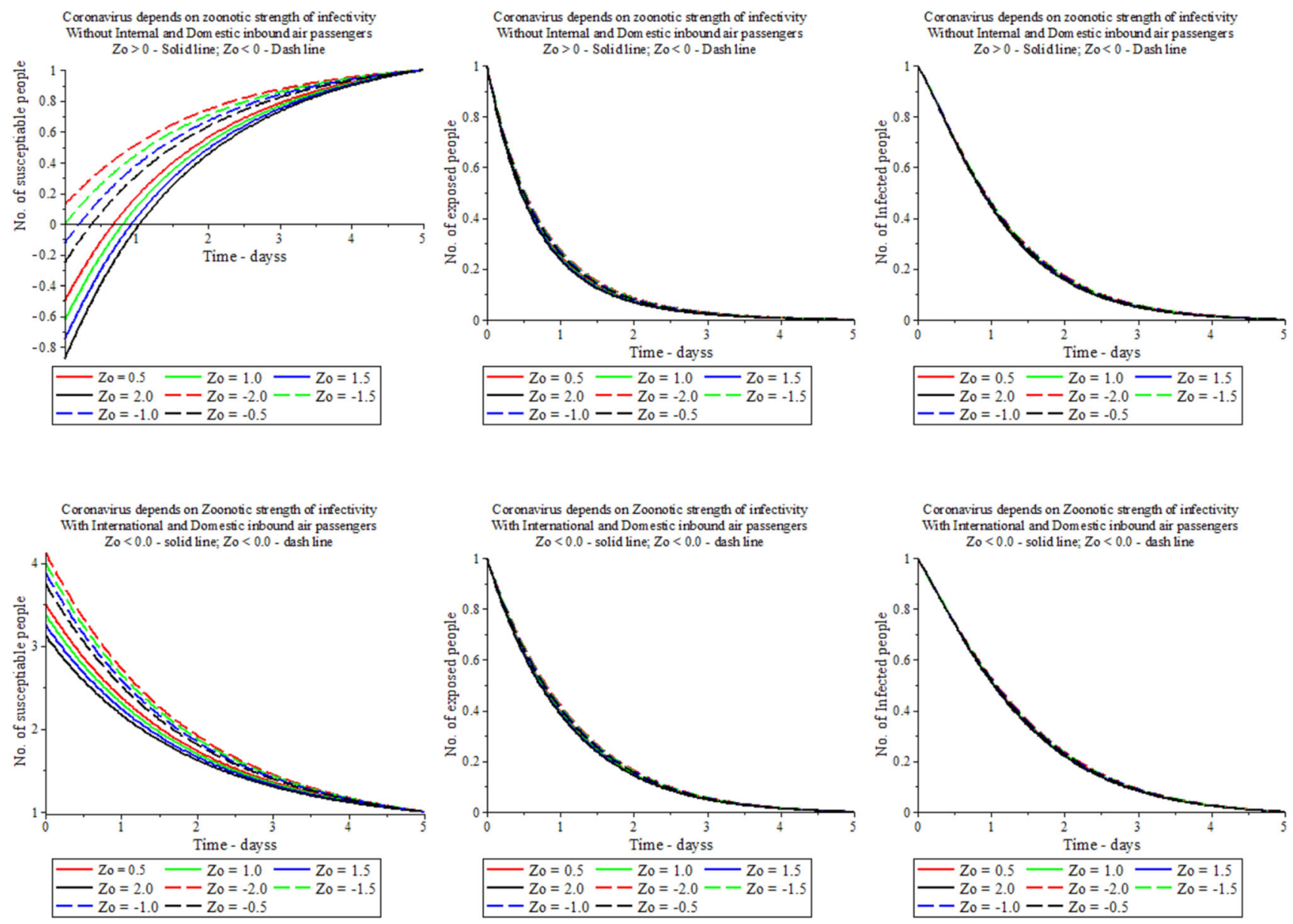

Fig. 4 Impact of zoonotic strength of infectivity on International and Domestic inbound air passengers 


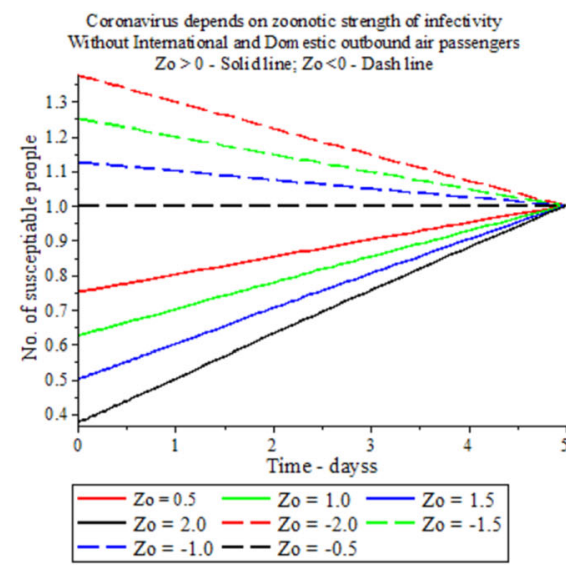

Cor onavirus depends on Zoonotic strength of infectivity With International and Domestic outbound air passengers

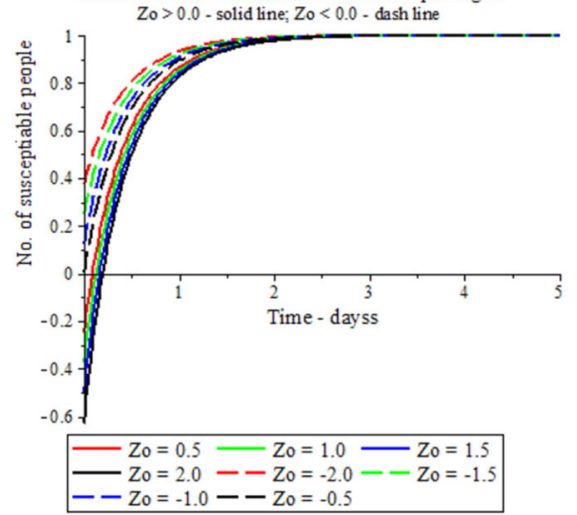

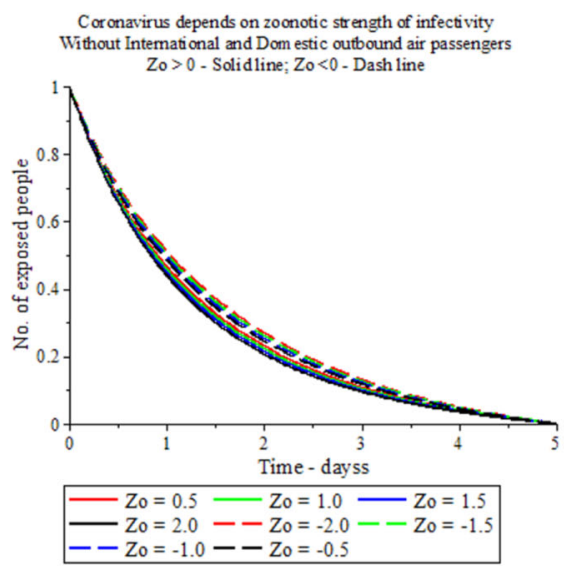

Cor onavirus depends on Zoonotic strength of infectivity ith International and Domestic outbound air passengers

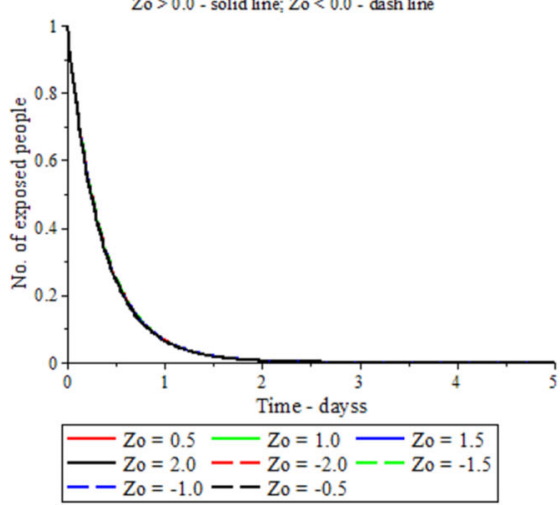

Cor onavirus depends on zoonotic strength of infectivity Without Intemational and Dom estic outbound air passengers

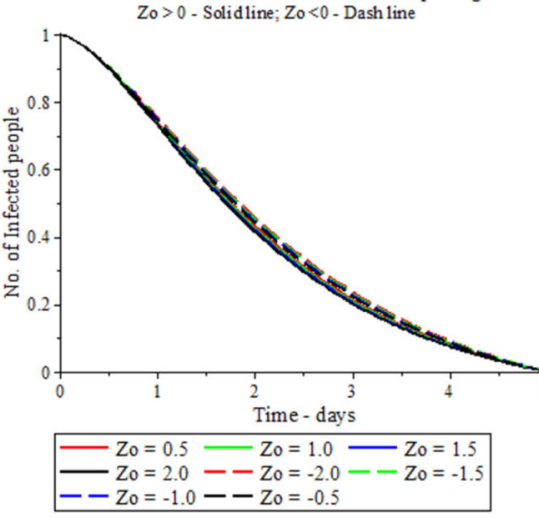

Cor onavirus depends on Zoonotic strength of irfectivity With International and Domestic outbound air passengers

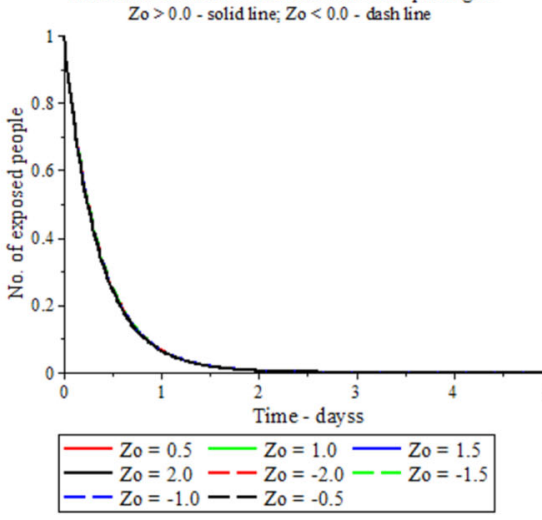

Fig. 5 Impact of basic reproductive strength on International and Domestic outbound and air passengers

power of contagion establishes the utmost aspect conceivable of COVID-19 infection. It expresses what may want to occur if an infectious person has been to access a completely prone, exposable indefectible public, and consequently it considers primarily based on a crucial occasion. The controlling zoonotic power of contagion depends on the population's leading-edge vulnerability. The green Zo modifications over the years to period and its miles, a control mostly constructed on workable atmosphere within the population. Virtually the front-runner sign of the COVID-19 depends on lungs inflexibility of breathing. All nations discovered that the zoonotic infectivity and reproductive number hits an important role for controlling the COVID-19 infections. With or without international and domestic inbound and outbound air passengers, the number of susceptible peoples rate of COVID-19 decreases whereas the exposed and infected peoples with rise of zoonotic strength of infectivity whereas the susceptible peoples density of $\mathrm{Zo}<0$ was more significant as compared to Zo $>0$.

International and Domestic inbound and outbound air travelers are the main factors for spreading coronavirus unusually fast. The system and subsequent coronavirus outbreaks are based on a shift in zoonotic infection powers. Our review focuses on the effectiveness the Zo to protect this meaning, Figs. 4 and 5. So, both international and domestic inbound and outbound air passengers have specified significant position to control coronavirus. Zoonotic is a transmissible complaint pretentious by a pathogen that has blowout from beasts to persons. But recently, coronavirus infectious spread from humans to humans extremely fast through direct physical contact, through the air or water. Constructed on this study, the SEIR people's rate slows down to widen of zoonotic power, although the SEIR people's rates condense as rising of zoonotic power of infectivity. In the presence of basic productive number, $R_{o}<1$, it is predicted that the susceptible, exposed and infected people's moves up and down whereas for $R_{o}>1$, the susceptible, exposed and infected peoples decelerates with the rise of zoonotic strength of corona infectivity, Fig. 6.

From Table 1, Figs. 6, and 7, the incidence of susceptible and exposed subjects increased and infected persons decreased with increased animal infection strength 
Fig. 6 Impact of basic reproductive strength on the zoonotic strength of coronavirus infectivity

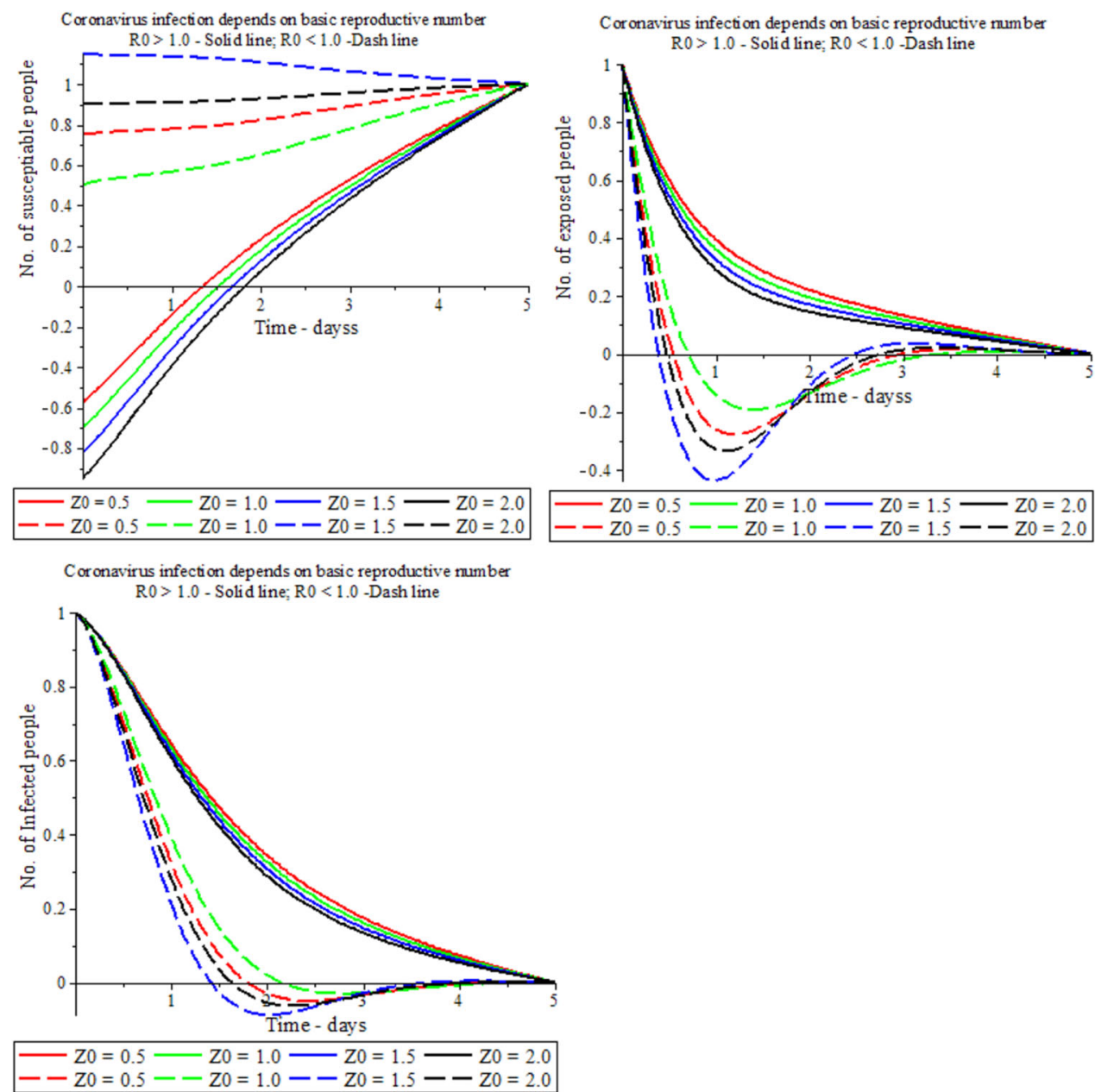

following the high baseline reproductive count, $\mathrm{Ro}=2$. If the basal reproductive value is small $(\mathrm{Ro}=-2.0)$, the susceptible person rises first and then decreases, while the exposed and infected individuals decrease first and then increase with the strength of the zoonotic infection, Table 1, Figs. 7 and 8. In the presence of zoonotic infectivity, it is observed that the exposed peoples from COVID19 were higher compared to the susceptible and infected peoples, 3D graph, Figs. 7 and 8. All countries are ready to take special steps to wear masks at shipping centers, seaports, and airports and border crossings to control the spread of COVID-19, the sum of the strength of the zoonotic infection and its number, basic reproduction plays a key role in the ultra-fast spread of COVID-19.

\section{Conclusion}

The basic reproductive number and zoonotic strength of infectivity on susceptible, exposed, and infected people's rate were reviewed engaging Runge-Kutta-Fehlberg approach through by MAPLE 18 .

- The Zoonotic infectivity from international and domestic, inbound or outbound air travelers are a key factor in the spread of the unusually fast COVID-19.

- With zoonotic infectivity, it was observed that those exposed peoples to COVID-19 were higher compared to those who were susceptible and infected peoples.

- In the presence of basic productive strength, $\mathrm{R}(\mathrm{o})<1$, it is predicted that the exposed peoples rate moves up and down with an increase of zoonotic infectivity. 

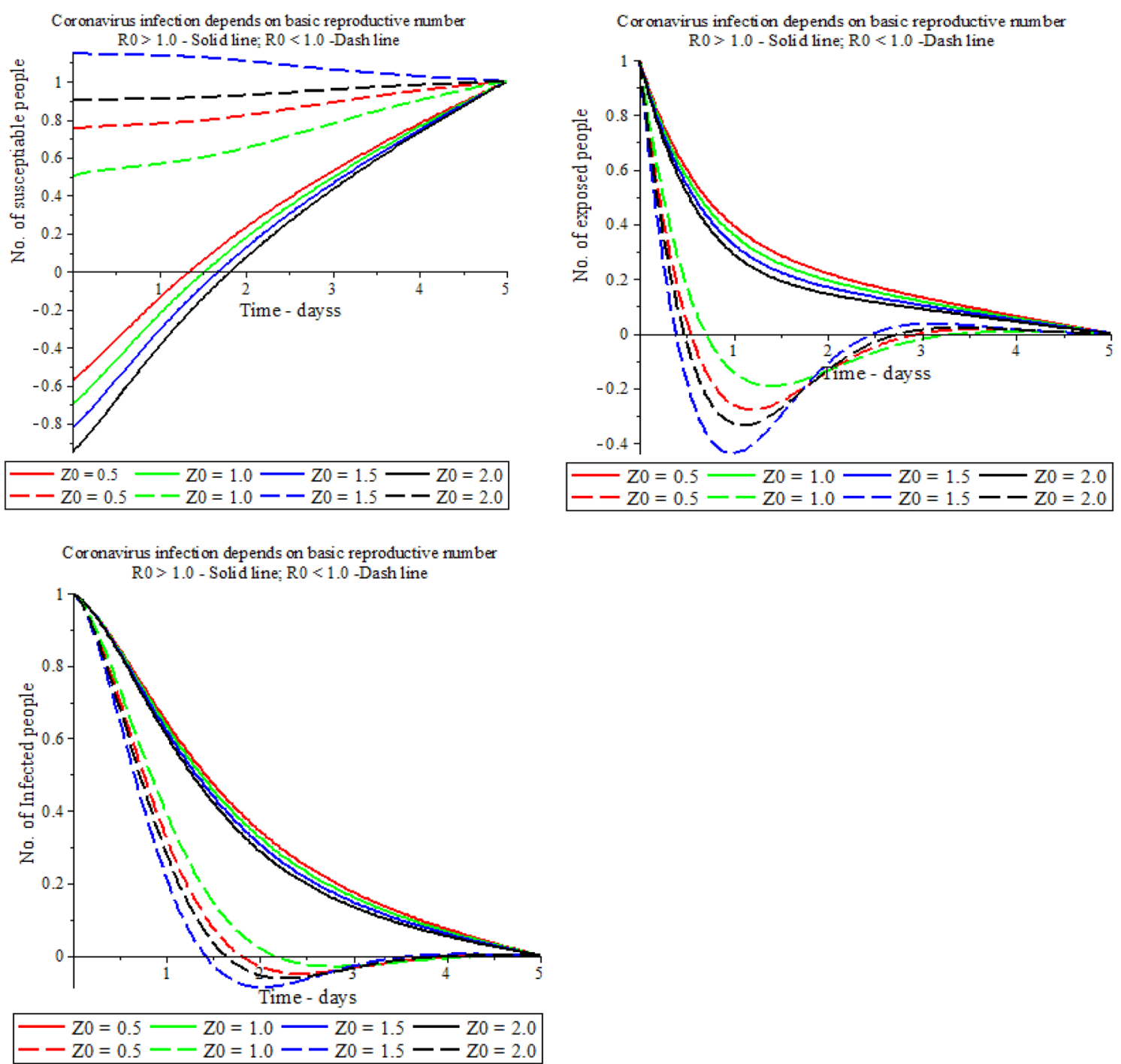

Fig. 7 Zoonotic infectivity on susceptible, exposed and infected peoples

- COVID-19 is a highly transmissible infection virus since it flows as of public to public extremely fast because of Zoonotic infectivity.
COVID-19 transmission from humans to humans fast through direct physical contact, through the air or water, has been controlled by the combined effect of the basic reproductive number and zoonotic strength of infectivity. 

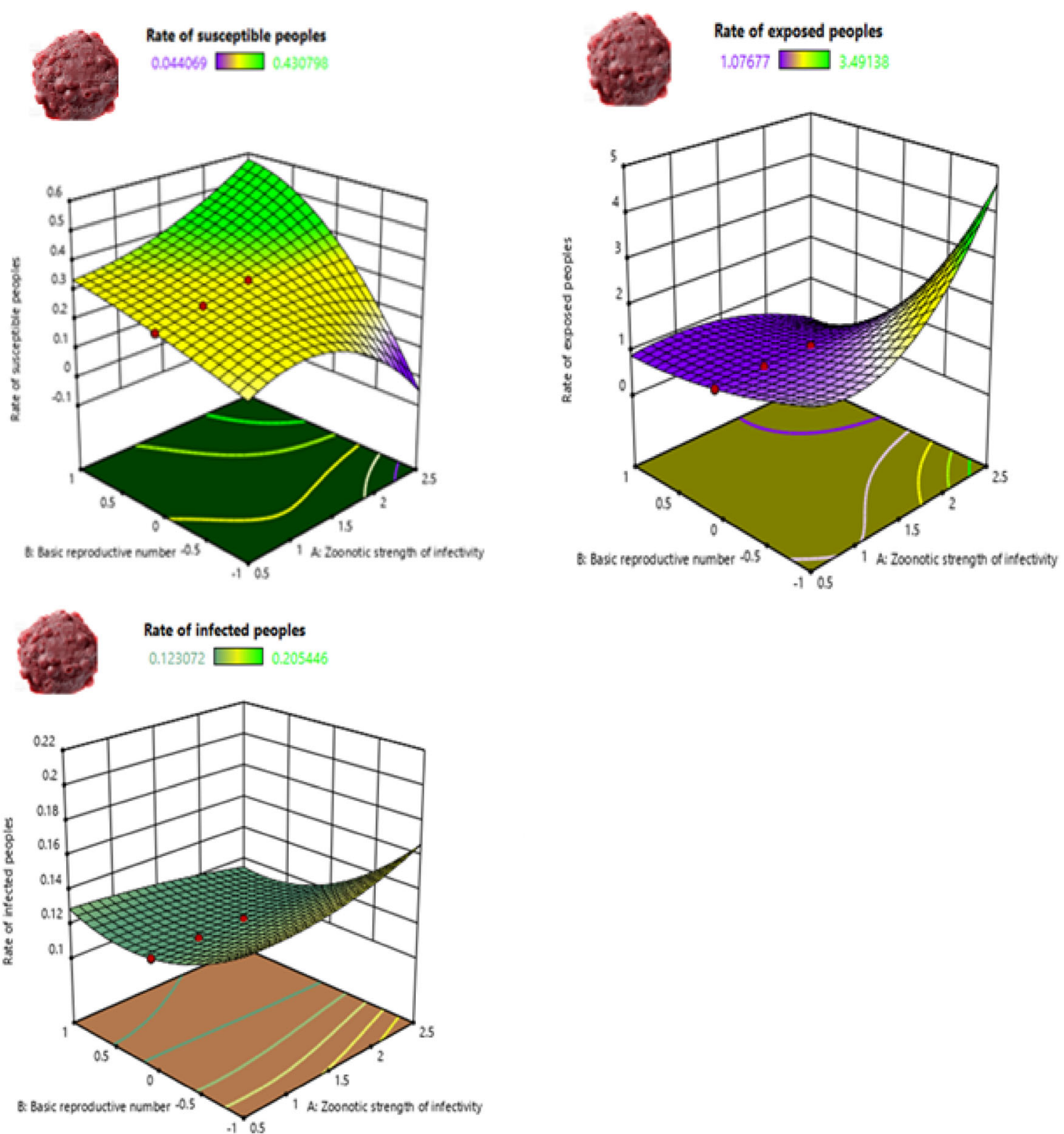

Fig. 8 Zoonotic strength of infectivity on coronavirus by statistical bar chart

Table 1 Rate of number of vulnerable, unprotected, and diseased peoples based on zoonotic strength of infectivity

\begin{tabular}{|c|c|c|c|c|}
\hline Zoono. strength Reproduct. of infect., Zo & Rate of susceptible peoples & Rate of exposed peoples & Rate of infected peoples & Basic number \\
\hline 0.5 & 0.378113 & 1.076774 & 0.16553 & R0 $(=2.0)>1$ \\
\hline \multicolumn{5}{|l|}{1} \\
\hline 1.0 & 0.404685 & 1.1568083 & 0.159179 & \\
\hline 1.5 & 0.430798 & 1.2392051 & 0.15298 & \\
\hline 0.5 & 0.27321 & 1.1045138 & 0.123072 & $\mathrm{R} 0(=0.0)$ \\
\hline 1.0 & 0.304979 & 1.1045138 & 0.123072 & \\
\hline 1.5 & 0.336747 & 1.1045138 & 0.123072 & \\
\hline 0.5 & 0.076931 & 2.7483809 & 0.201877 & R0 $(=-2.0)<1$ \\
\hline \multicolumn{5}{|l|}{$<1$} \\
\hline 1.0 & 0.157887 & 2.3244739 & 0.199087 & \\
\hline 1.5 & 0.044069 & 3.491377 & 0.205446 & \\
\hline
\end{tabular}

Bold represents rate of number of vulnerable, unprotected, and diseased peoples based on zoonotic strength of infectivity 
Acknowledgements The authors encompass their obligation to The President, Vice President Academic, and Dean of Natural Science, Salale University, Fiche, Oromia Region, Ethiopia.

\section{References}

1. World Health Organization. Coronavirus (2020). https://www.who.int/health-topics/coronavirus.

2. Zhou P, Yang XL, Wang XG, Hu B, Zhang L, Zhang W et al (2020) A pneumonia outbreak associated with a new coronavirus of likely bat origin. Nature. https://doi.org/10.1038/s41586020-2012-7

3. Li Q, Guan X, Wu P, Wang X, Zhou L, Tong Y et al (2020) Early transmission dynamics in Wuhan, China, of novel coronavirusinfected pneumonia. The New Engl J Med. https://doi.org/ 10.1056/NEJMoa2001316

4. Zhu N, Zhang D, Wang W, Li X, Yang B, Song J et al (2020) A novel coronavirus from victims with pneumonia in China 2019. The New Engl J Med. https://doi.org/10.1056/NEJMoa2001017

5. Huang C, Wang Y, Li X, Ren L, Zhao J, Hu Y et al (2020) Clinical factors of victims contaminated with 2019 novel coronavirus in Wuhan. Lancet. https://doi.org/10.1016/S01406736(20)30183-5

6. Chan JF et al (2020) A familial cluster of pneumonia associated with the 2019 novel coronavirus indicating personto-person transmission: a discover out about of a family cluster. Lancet. https://doi.org/10.1016/S0140-6736(20)30154-9

7. Bogoch II, Watts A, Thomas-Bachli A, Huber C, Kraemer MUG, Khan K (2020) Pneumonia of unknown etiology in Wuhan, China: potential for international spread via commercial air travel. J Travel Med. https://doi.org/10.1093/jtm/taaa008

8. Wu JT, Leung K, Leung GM (2020) Nowcasting and forecasting the viable domestic and international unfold of the 2019-nCoV outbreak originating in Wuhan, China: a modelling study. Lancet. https://doi.org/10.1016/S0140-6736(20)30260-9

9. Zhao $S$ et al (2020) Preliminary estimation of the easy replica volume of novel coronavirus (2019-nCoV) in China, from 2019 to 2020: a data-driven comparison in the early phase of the outbreak. Int $J$ Inf Dis. https://doi. org/10.1016/j.ijid.2020.01.050

10. Zhao S, Musa SS, Lin Q, Ran J, Yang G et al (2020) Estimating the unreported range of novel coronavirus (2019-nCoV) situations in China in the first half of of January 2020: a data-driven Modelling assessment of the early outbreak. J Clinic Med. https://doi.org/10.3390/jcm9020388

11. World Health Organization. Novel Coronavirus - China (2020) World Health Organization. https://www.who.int/csr/ don/12january-2020-novel-coronavirus-china/en/. Accessed 20 Jan 2020.

12. Chen T, Rui J, Wang Q, Zhao Z, Cui J-A, Yin L (2020) A mathematical model for simulating the transmission of Wuhan novel Coronavirus. bioRxiv., 2020: 2020.2001.2019.911669.

13. Chen T-M, Rui J, Wang Q-P, Zhao Z-Y, Cui J-A, Yin L (2020) A mathematical model for simulating the phase-based transmissibility of a novel coronavirus. Infect Dis Poverty. https:// doi.org/10.1186/s40249-020-00640-3

14. Wu JT, Leung K, Leung GM (2020) Nowcasting and forecasting the manageable domestic and international unfold of the 2019-nCoV outbreak originating in Wuhan. China: a modelling study. Lancet 395:689-697

15. Ye Z-W, Yuan S, Yuen K-S, Fung S-Y, Chan C-P, Jin D-Y (2020) Zoonotic origins of human coronaviruses. Int J Biol Sci 16(10):1686-1697

Publisher's Note Springer Nature remains neutral with regard to jurisdictional claims in published maps and institutional affiliations. 